B-to-A subproblems of A-2, A-3, and A-6) may be more sensitive to hypothyroid deficit (note the corresponding A-to-B subproblems) than to difficult symmetrical problems.

\section{MANUAL USE OF THE APPARATUS}

Essentially the same procedures we have described for this automated apparatus can be carried out manually, with most of the advantages over older closed-field test methods preserved and at a considerable saving of equipment (automatic doors, photocells, programming modules, and closed-circuit television). With manually operated guillotine doors and electrically operated pellet dispensers, an E could administer trials and record errors from a single (visually shielded) position near the apparatus without handling Ss between trials. Although we have no experience to report with such an arrangement, it would seem to be nearly equal to our automated version in convenience, accuracy of recording errors, $E$ influences, and applicability to species that are difficult to handle between trials. Except for the possibility that the low-level noise of the door motors may provide facilitative directional cues to $\mathrm{Ss}$ in the automated setting, there seems to be no good reason to expect any important differences between the automated and manual versions of the apparatus in the data obtained.

\section{REFERENCES}

DAVENPORT, J. W. Cretinism in rats: Enduring behavioral deficit induced by tricyanoaminopropene. Science, in press.

EAYRS, J. T. Functional correlates of modified cortical structure. In D. B. Tower and J. P. Shade (Eds.), Structure and function of the cerebral cortex. Amsterdam: Elsevier, 1960. Pp. 43-50.

HADDAD, R. K., RABE, A., LAQUEUR, G. L.
SPATZ, M., \& VALSAMIS, M. P. Intellectual deficit associated with transplacentally induced microcephaly in the rat. Science, $1969,163,88-90$

HEBB, D. O., \& WILLIAMS, K. A method of rating animal intelligence. Journal of Genetic Psychology, 1946, 34, 59-65.

LIVESEY, $P$. J. The rat, rabbit, and cat in the Hebb-Williams closed field test of animal intelligence. Australian Journal of Psychology, 1966, 18, 71-79.

RABINOVITCH, M. S., \& ROSVOLD, H. E. A closed field intelligence test for rats. Canadian Joumal of Psychology, 1951, 5, 122-128.

TRYON, R. C. Genetic differences in maze learning in rats. In National Society for the Study of Education, The thirty-ninth yearbook. Bloomington, Ill:: Public School Publishing Co., 1940. Pp. 111-119.

\section{NOTE}

1. Supported by Grant FR-0167 from the National Institutes of Health. We thank Will Retzlaff and Thomas Sorenson for assistance in testing the animals, and Sue Engelke and Diane Eich for statistical assistance.

\title{
A runway for the cockroach ${ }^{1}$
}

\author{
NICHOLAS LONGO, COLGATE \\ UNIVERSITY, Hamilton, New York \\ 13346
}

A runway and training procedure that minimizes handling is described. Acquisition and extinction trials are presented to illustrate the technique.

Instrumental learning in cockroaches has been studied under escape-avoidance conditions (Longo, 1964), but no suitable food-reward procedure yet has been described in the literature, although it would be useful to have such a procedure for purposes of phyletic comparison.

Two problems have been encountered in our attempt to develop a food-reward procedure. One is that the appetite of the cockroach is not large enough. Minute quantities of food and water are sufficient to maintain the animals in a healthy, active state. The common roaches, Periplaneta americana and $P$. orientalis, have lived as long as 2 months without any food and water in my laboratory. The solution to this problem is to use a highly preferred food, such as canned crushed-andsweetened pineapple. A second problem is that cockroaches, like fishes (Potts \& Bitterman, 1968), are disturbed by handling. In earlier experiments, I attempted to minimize this difficulty by cementing a loop of nylon thread to the thorax of the animal and carrying it by the loop, but roaches are reluctant to enter a

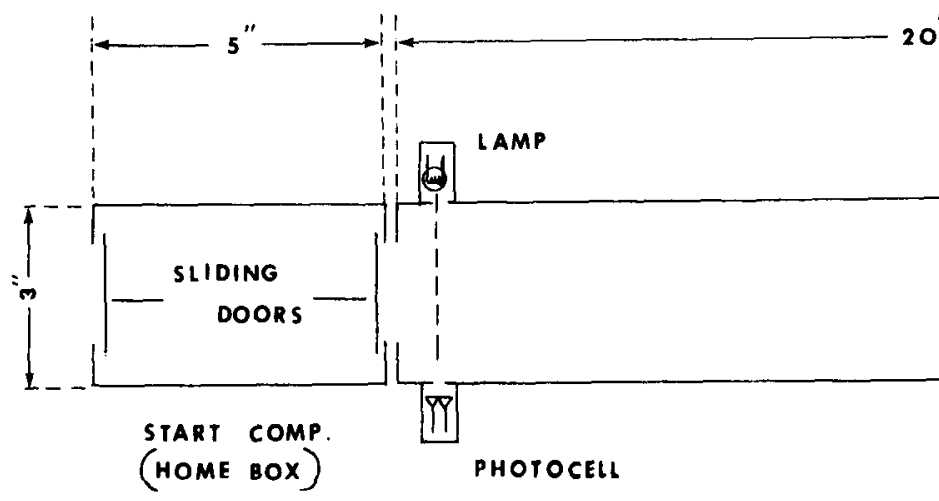

Fig. 1. A runway for cockroaches. The start and goal compartments are interchangeable. Food is introduced into the goalbox through the sliding door at the back.

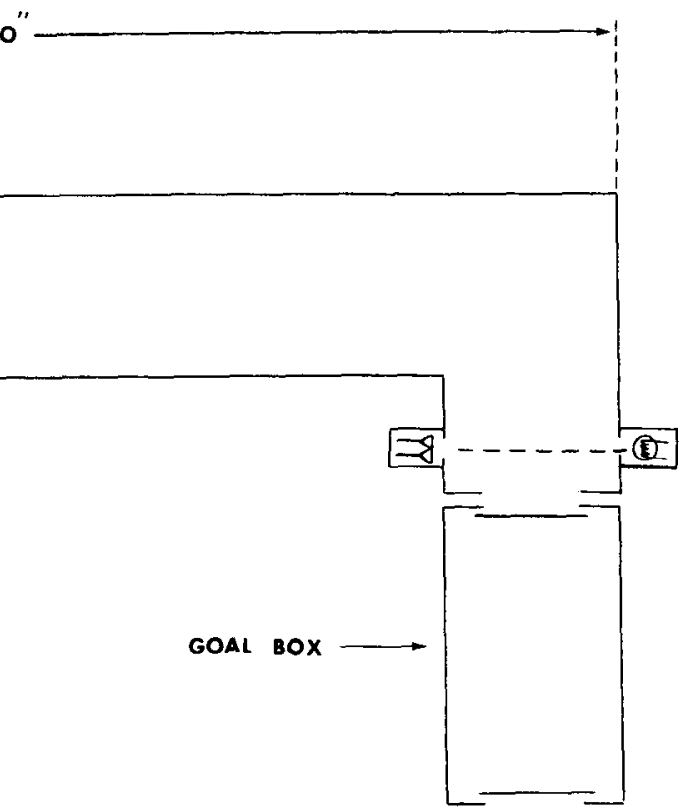


Fig. 2. The course of acquisition and extinction in the runway.

goal compartment from which they are removed in this way. The solution to this problem is to carry the animal in its living compartment.

The runway diagrammed in Fig. 1 has interchangeable start and goal compariments which serve also as living compartments. These compartments, constructed of 1/8-in. black Plexiglas and equipped with sliding doors, are $5 \times 3 \times 2.5 \mathrm{in}$. high. The plastic runway is $20 \times 3 \times 2.5$ in. high. Photocells and light sources are mounted at each end of the runway to measure latency and running time. The compartment containing the experiniental animal is brought to the front of the runway, and an identical empty box is placed at the other end. A trial is begun by lifting the sliding door of the living chamber and depressing a switch to turn on the latency timer. When the roach leaves the home compartment, it breaks the first photobeam, stopping the latency clock and activating a second clock that measures running time. The sliding door to the goal compartment is lowered after the animal enters, and it is allowed to eat for $10 \mathrm{sec}$ from a food tray inserted through the sliding door at the rear. Then the food is withdrawn, and the goal compartment containing the animal is removed from the end of the runway.

The course of acquisition and extinction with a sample of 10 roaches of the species Byrsotria fumigata (Guérin) is plotted in Fig. 2 in terms of mean log latency and mean $\log$ running time. After 7 days of adaptation to the individual compartments, during which time they were deprived entirely of food and water, the animals were given four trials for 30 days. All the animals were trained in groups of five, making the intertrial interval for each dependent on the latency and running time of the group; on the average, the interval

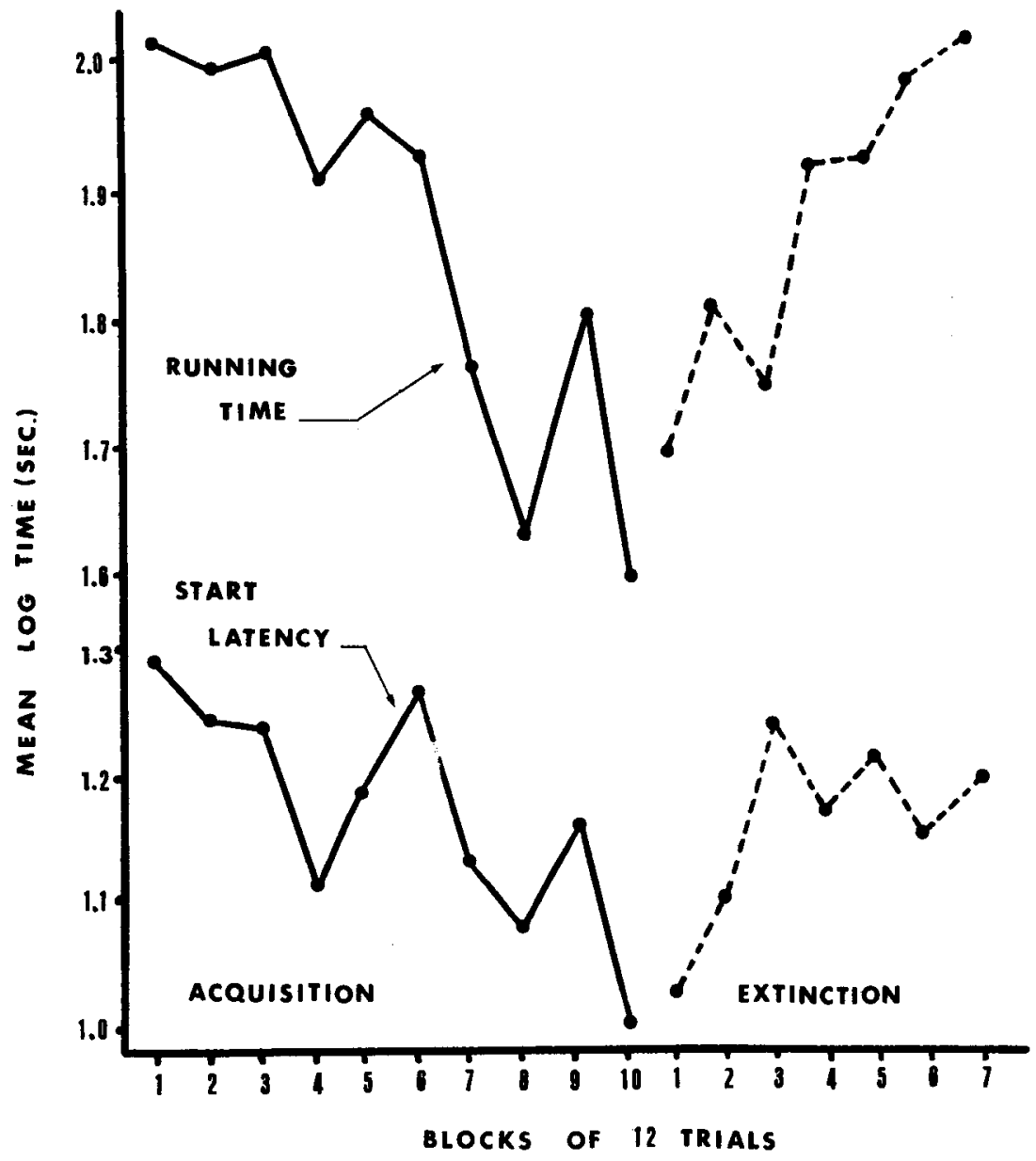

was about $7 \mathrm{~min}$. All trials were conducted in a room illuminated only by a 7 -W lamp in one corner and the small amount of light from the photocell lamps. After 30 days of consistent reinforcement with $10-\mathrm{sec}$ access to pineapple on each trial, performance had stabilized and extinction was begun. On extinction trials, the animal was removed (in its goal compartment) $15 \mathrm{sec}$ after it entered that compartment. The curves of Fig. 2 are characteristic of those found in higher animals.

\section{REFERENCES}

LONGO, N. Probability-learning and habit-reversal in the cockroach. American Journal of Psychology, 1964, 77, 29-41.

POTTS, A., \& BITTERMAN, M. E. A runway for the fish. Behavior Research Methods \& Instrumentation, 1968, 1, 26-27.

\section{NOTE}

1. This research was supported by Grant MH 10846-3 from the Public Health Service and a grant from Carter-Wallace, Inc. The author thanks Dr. Louis M. Roth of the U.S. Army Natick Laboratories for supplying the experimental animals. 